\title{
DETERMINAÇÃO DO CONSUMO DE FOLHAS DE Eucalyptus grandis POR FORMIGUEIROS DE Atta sexdens rubropilosa
}

\author{
Daniele Ukan ${ }^{1}$, Nilton José Sousa ${ }^{2}$, Claudiane Belinovski ${ }^{3}$ \\ ${ }^{1}$ Eng $^{\mathrm{a}}$ Florestal, Dra ${ }^{\mathrm{a}}$, Depto. de Engenharia Florestal, UNICENTRO, Irati, PR, Brasil - daniukan@ yahoo.com.br \\ ${ }^{2}$ Eng. Florestal, Dr., Depto. de Ciências Florestais, UFPR, Curitiba, PR, Brasil - nilton@ufpr.br \\ ${ }^{3}$ Acadêmica do curso de Eng. Florestal, UFPR, Curitiba, PR, Brasil - claudianeclpr@yahoo.com.br \\ Recebido para publicação: 09/04/2012 - Aceito para publicação: 03/10/2013
}

\begin{abstract}
Resumo
Pelo fato de cortarem material vegetal e o transportarem para o interior de suas colônias, as saúvas tornam-se importantes agentes de acumulação de matéria orgânica no solo, por meio de suas câmaras de descarte (lixo). Esse material acumulado é oriundo do processamento do fungo simbionte e se torna uma fonte de nutrientes. Diante desse contexto, o objetivo do trabalho foi determinar a conversão de folhas de Eucalyptus grandis em resíduos, por colônias de Atta sexdens rubropilosa monitoradas em laboratório. Foram selecionadas 8 colônias de Atta sexdens rubropilosa, sendo 4 coletadas em 2007 (denominados adultos) e 4 coletadas em 2010 (denominadas jovens). Durante 72 dias, elas foram supridas com folhas de mudas de Eucalyptus grandis. A média do fator de conversão entre os formigueiros adultos e jovens monitorados em laboratório foi de 1,49. Observou-se que $63,65 \%$ do material fornecido aos formigueiros converteram-se em resíduos.

Palavras-chave: Resíduos; formigas-cortadeiras; taxa de conversão.
\end{abstract}

\begin{abstract}
Determination of consumption of Eucalyptus grandis by nests of Atta sexdens rubropilosa. Because ants cut and carry plant material inside their colonies, they become important agents for the accumulation of organic matter in the soil through their disposal chambers. This accumulated material comes from symbiotic fungus processing and can become a source of nutrients. In the context, the objective of this study was to determine the conversion of leaves of Eucalyptus grandis into waste by colonies of Atta sexdens rubropilosa monitored in laboratory. We selected eight colonies of Atta sexdens rubropilosa, 4 collected in 2007 (called adults), and 4 in 2010 (referred to as young). During 72 days, they were supplied with leaves of Eucalyptus grandis. The average conversion factor between the young and adult colonies monitored in the laboratory was 1.49 . We observed that $63.65 \%$ of the material supplied to the colonies converted to waste.

Keywords: Waste; leaf-cutting ants; conversion rate.
\end{abstract}

\section{INTRODUÇÃO}

Pelo fato de cortarem material vegetal e o transportarem para o interior de suas colônias, as saúvas tornam-se importantes agentes de acumulação de matéria orgânica no solo, por meio de suas câmaras de descarte (lixo). Esse material acumulado é oriundo do processamento do fungo simbionte e pode se tornar uma fonte de nutrientes, sendo possível sua reabsorção pelas raízes das plantas por meio da ciclagem biogeoquímica de elementos (MOUTINHO et al., 2003; VERCHOT et al., 2003).

$\mathrm{O}$ acúmulo no subsolo de matéria orgânica do material vegetal cortado e incorporado ao fungo simbionte, bem como o material exaurido e carcaças de formigas mortas (lixo) modificam os solos, por aumentar a concentração de nutrientes liberados durante o processo de decomposição da matéria orgânica. Contudo, a determinação do dano por herbivoria e a quantidade de material vegetal acumulado no interior das colônias dessas espécies é alvo de controvérsias (AUTUORI, 1947; JONKMAN, 1977; FOWLER et al., 1990).

O cálculo da taxa de conversão é uma forma de avaliação do dano da herbivoria, dividindo-se a massa (em gramas) do material cortado pelo resíduo produzido (AUTUORI, 1947). Utilizando-se esse método, as taxas de conversão de Atta vollenweideri Forel e Acromyrmex landolti Forel (Hymenoptera: Formicidae) foram estimadas em 1,5 e 1,8, respectivamente (JONKMAN, 1977). Entretanto, a qualidade 
do material cortado pode influenciar a produção de lixo (WEBER, 1976). Nesse caso, se a qualidade do substrato afeta essa produção, é possível que ela também altere as taxas de conversão das diferentes espécies de formigas cortadeiras (SOUTO et al., 2007).

Souto et al. (2007) registram que as colônias de Atta sexdens rubropilosa tendem a aumentar o consumo de material vegetal de baixa qualidade. A taxa de conversão dessa espécie é de aproximadamente 1,5, independentemente da qualidade do material forrageado.

Recentes estudos têm demonstrado que o fungo simbionte cultivado pelas formigas-cortadeiras não é eficiente em degradar celulose e lignina (ABRIL; BUCHER, 2002; 2004), reforçando a hipótese de que apenas, 11-27\% do material vegetal incorporado ao jardim de fungo seja metabolizado (ROBBINS, 1993).

Diante desse contexto, os objetivos deste trabalho foi determinar a conversão de folhas de Eucalyptus grandis em resíduos, por colônias de A. sexdens rubropilosa monitoradas em laboratório.

\section{MATERIAL E MÉTODOS}

Os materiais analisados foram amostras de mudas e folhas de Eucalyptus grandis utilizadas para suprir os formigueiros e os resíduos produzidos pelas colônias.

\section{Formigueiros utilizados nos experimentos}

O material biológico, ou seja, os formigueiros utilizados nos experimentos, foram coletados nas áreas de ocorrência natural de A. sexdens rubropilosa (saúva-limão), nos estados do Paraná e São Paulo, em plantios de $E$. grandis, cerca de três meses após a revoada, que ocorre normalmente entre outubro e novembro. Foram coletadas 105 colônias em fevereiro de 2007 e 80 em fevereiro de 2010, a fim de garantir um número mínimo de sobrevivência das colônias para a realização dos experimentos.

A realização da coleta, cerca de três meses após a revoada, está baseada em relatos biológicos de autores como Autuori (1947) de que nesse período os formigueiros sobreviventes já estão estabelecidos e encontram-se em uma profundidade média de $13,4 \mathrm{~cm}$.

Foram percorridos os talhões com plantio de E. grandis onde a coleta das colônias eram realizadas no começo da manhã e ao entardecer, devido a alterações de umidade, sendo identificados os ninhos da espécie $A$. sexdens rubropilosa nas linhas e entrelinhas dos mesmos. Com o auxílio de um enxadão, foram feitas trincheiras laterais aos ninhos, para que a colônia fosse retirada com mínimas perdas (Figura 1), tanto do fungo quanto das formigas e principalmente da rainha.

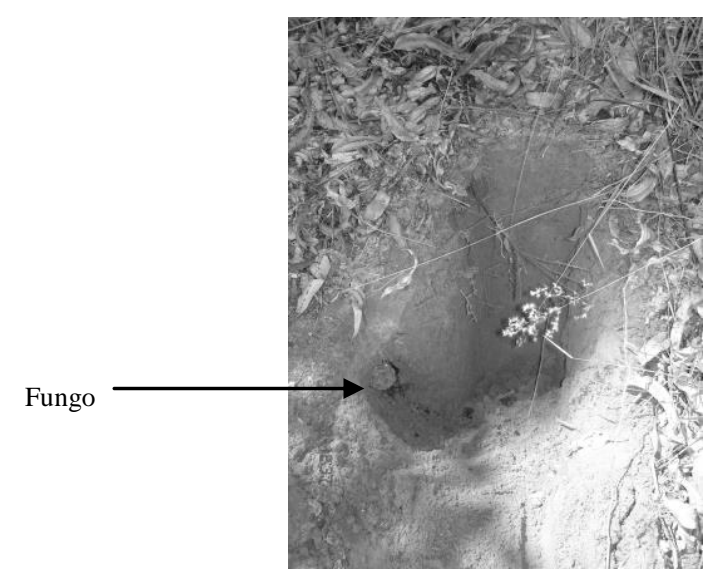

Figura 1. Coleta de formigueiros três meses após a revoada. Trincheira aberta para a retirada do fungo. Botucatu, SP.

Figure 1. Collection of anthills three months after flight. Trench opened to remove the fungus.

Após a abertura e visualização do ninho, com auxílio de uma colher, foram retirados todos os constituintes da colônia, que foram armazenados em recipientes plásticos $(250 \mathrm{~mL})$, com uma camada de $\pm 2 \mathrm{~cm}$ de gesso na base, para conservação e umidade das colônias, conforme procedimentos práticos para as coletas, obtidos em conversas informais com o Professor Dr. Luiz Carlos Forti (2007) (Figura 2). 


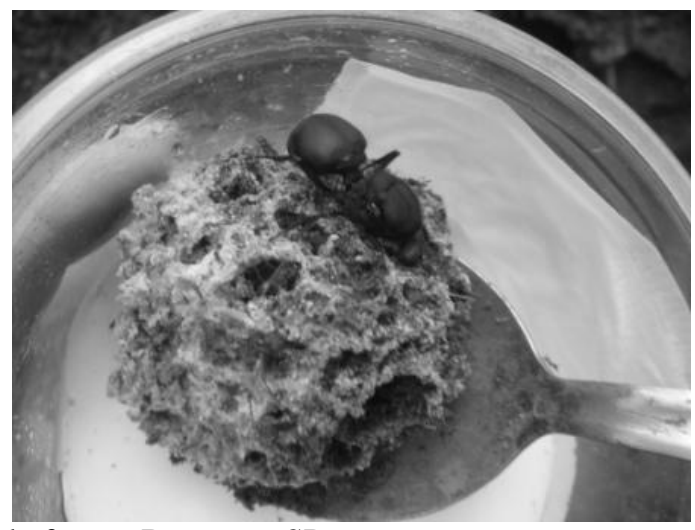

Figura 2. Armazenamento do fungo. Botucatu, SP.

Figure 2. Storage of the fungus. Botucatu, SP.

Concluída a coleta, os ninhos foram armazenados e transportados para o Laboratório de Proteção Florestal da UFPR em caixas de isopor, para evitar a perda de umidade, devido à exposição à luz solar.

No laboratório, o material coletado foi acondicionado em uma sala com condições controladas de temperatura e umidade, $\pm 25^{\circ} \mathrm{C}$ e $70 \%$ de UR, respectivamente (Figura 3). Para impedir a entrada de raios ultravioletas (UVA), os vidros das janelas da sala foram isolados com papel alumínio, sendo utilizadas lâmpadas fluorescentes para sua iluminação.

Após a coleta, as colônias permaneceram por três meses num período de estabilização, sendo alimentadas com flores e folhas de rosas (Rosa x grandiflora Hort.), alfeneiro (Ligustrum lucidum), robinia (Robinia pseudoacacia), amora (Morus nigra) e duas espécies de eucaliptos (E. grandis e Eucalyptus dunnii).

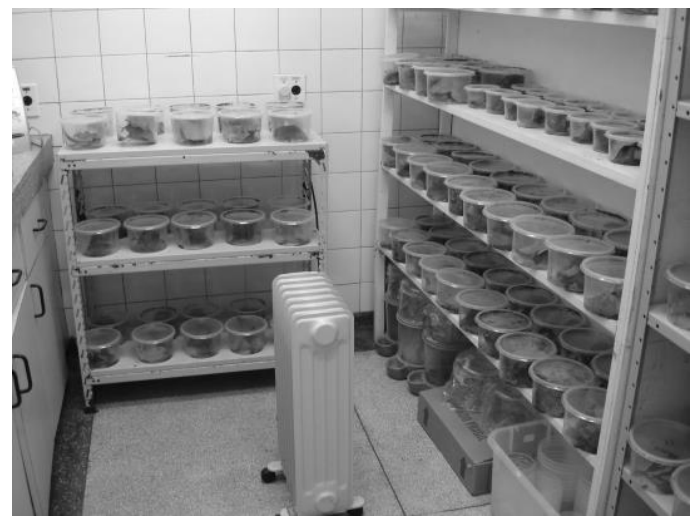

Figura 3. Monitoramento de formigueiros em laboratório. UFPR, Curitiba, PR.

Figure 3. Monitoring of colonies in laboratory. UFPR, Curitiba, PR.

Dentro do período de dois meses depois da coleta, quando as colônias começaram a se desenvolver e ocupar praticamente todo o recipiente menor $(250 \mathrm{~mL})$, elas foram alocadas em recipientes maiores $(500 \mathrm{~mL})$ e ligadas a um recipiente menor por uma mangueira de plástico de $\pm 2 \mathrm{~cm}$ de diâmetro, destinada a ser a panela de resíduo, padrão esse ocorrido de forma regular para criação de formigueiros em laboratório (Figura 4).

\section{Suprimento das colônias com folhas de Eucalyptus grandis}

As colônias selecionadas para o experimento passaram a ser supridas com Eucalyptus grandis, por ser esta uma espécie florestal com alto nível de corte/desfolhamento pelos formigueiros de A. sexdens rubropilosa em campo e pela facilidade de adquirir mudas dessa espécie. 


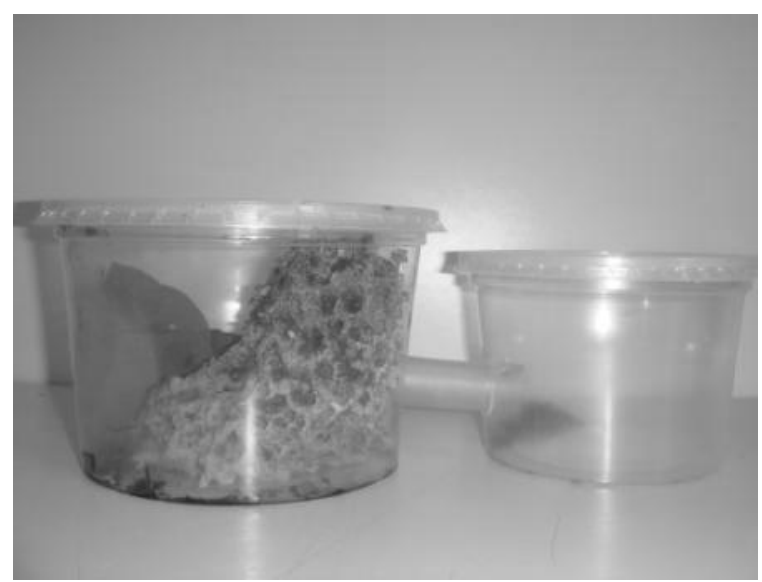

Figura 4. Recipiente para o desenvolvimento do formigueiro. Curitiba, PR.

Figure 4. Recipient for development of the colony. Curitiba, PR.

Experimentação e determinação da taxa de conversão de folhas de Eucalyptus grandis em resíduos

Foram selecionadas oito colônias de A. sexdens rubropilosa, sendo quatro coletadas em 2007 (denominadas adultas) e quatro coletadas em 2010 (denominadas jovens). Tanto no grupo de colônias jovens quanto na de adultos, foram selecionadas as colônias com o mesmo padrão de desenvolvimento (tamanho do fungo analisado visualmente). Dessa forma, o corte das espécies vegetais, a princípio, ocorreu de forma proporcional em todos os formigueiros.

Escolhidas as colônias, durante 72 dias elas foram supridas com folhas de mudas de E. grandis com aproximadamente 90 dias, doadas por um viveiro florestal da Região Metropolitana de Curitiba, chamado Itrópica Viveiro de Mudas. Antes do fornecimento das folhas para as colônias, elas eram pesadas em balança analítica e, na troca da alimentação no dia seguinte, o material não consumido era pesado novamente. Assim, obteve-se a quantidade de folhas que foram consumidas a cada 24 horas por cada colônia. Esses valores de consumo foram utilizados para a obtenção da taxa de conversão (relação da massa do material cortado pelo resíduo produzido).

Semanalmente eram coletados os resíduos gerados por cada colônia. Eram pesados (temperatura $\pm 25^{\circ} \mathrm{C}$ e umidade entre 60 e $80 \%$, dados de laboratório) e, por diferença, era contabilizada a quantidade de material que entrou na colônia (folhas de eucalipto) e a quantidade que saiu na forma de resíduo (Figura 5). A taxa de conversão (AUTUORI, 1947) foi obtida entre os 30 e 72 dias do experimento, dividindo-se a massa do material cortado pelo resíduo produzido. Assim, pode-se obter a proporção dos nutrientes das folhas consumidas pelo fungo e a colônia e o volume de resíduo gerado.

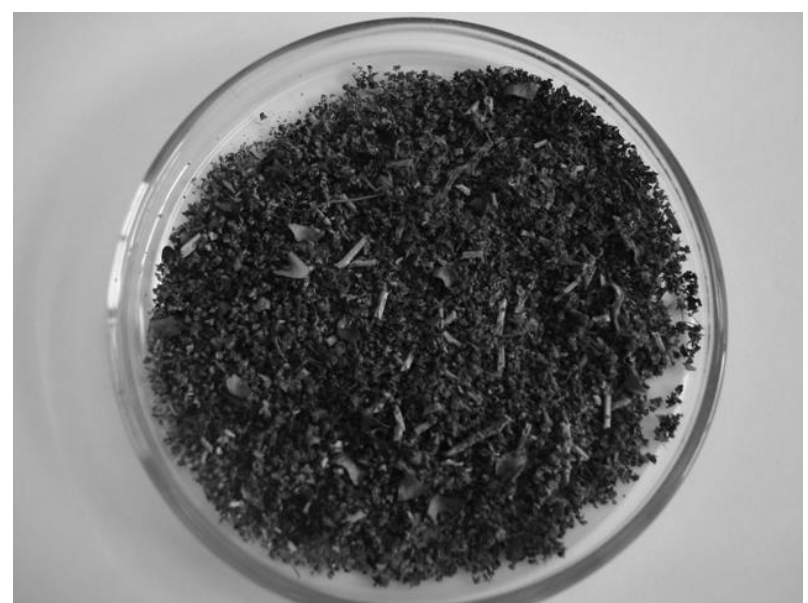

Figura 5. Resíduo de Atta sexdens rubropilosa. Curitiba, PR, BR.

Figure 5. Waste of Atta sexdens rubropilosa. Curitiba, PR, BR. 


\section{Análise estatística}

Procedeu-se à análise estatística utilizando-se o Software Assistat 7.5 Beta (SILVA; AZEVEDO, 2002). Realizou-se o teste Bartlett para verificar a homogeneidade das variâncias, a análise das variâncias (ANOVA) para a comparação de médias e o teste de Tukey ao nível de 5\% de probabilidade.

\section{RESULTADOS E DISCUSSÃO}

Durante o experimento, as colônias de formigas adultas cortaram mais folhas e, consequentemente, depositaram mais resíduos que as colônias de formigas jovens (comparação por meio de análise visual). As médias de consumo estão na tabela 1.

Tabela 1. Consumo total de folhas de Eucalyptus grandis (matéria seca) pelas colônias de Atta sexdens rubropilosa durante 42 dias.

Table 1. Total consumption of leaves of Eucalyptus grandis (dry matter) by colonies of Atta sexdens rubropilosa during 42 days.

\begin{tabular}{lc}
\hline Tratamento & Consumo total $(\mathbf{g})$ \\
\hline 1 - Colônia de formiga adulta & $103,12 \mathrm{a}$ \\
2 - Colônia de formiga jovem & $14,28 \mathrm{~b}$ \\
QME & $18.614,265$ \\
$\mathrm{~F}$ & $84,7729 * *$ \\
$\mathrm{CV}$ & 23,245 \\
\hline **: significativo ao nível de 1\% de probabilidade $(\mathrm{p}<01)$.**: Significant at $1 \%$ probability $(\mathrm{p}<01)$.
\end{tabular}

Verificou-se, nos formigueiros adultos, uma média de consumo de 103,12 gramas, e nos jovens, de 14,28 g, em 42 dias (Figura 6). Essas médias são diferentes estatisticamente em um nível de 1\% de probabilidade. Extrapolando esses valores para o período de um ano, o consumo por colônias adultas seria de aproximadamente $900 \mathrm{~g}$ e para as colônias jovens de $124 \mathrm{~g}$. Esses valores foram extrapolados para um consumo médio anual, desconsiderando-se as estações do ano.

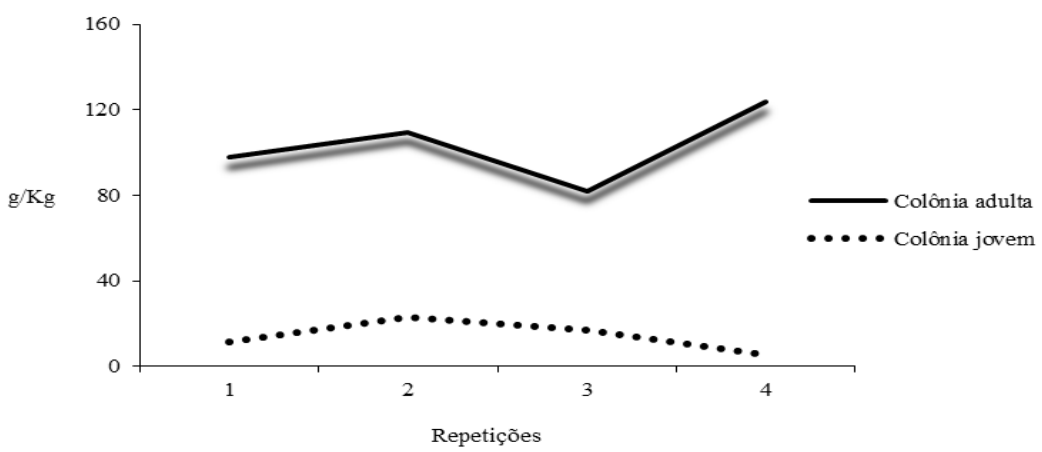

Figura 6. Consumo realizado pelo fungo simbionte de Atta sexdens rubropilosa.

Figure 6. Consumption by the symbiotic fungus of Atta sexdens rubropilosa.

A princípio, esses valores parecem pequenos, porém, considerando-se que no campo os formigueiros são maiores, chegando a apresentar mais de $100 \mathrm{~m}^{2}$ de área (FORTI et al., 1987), esses valores podem ser expressivos, pois o número de operárias (média \pm 1.500 ) das colônias em laboratório foi pequeno, comparado ao de colônias adultas dessa espécie em habitat natural. Forti (2008), escavando um formigueiro de $A$. sexdens rubropilosa, estimou que pudessem ocorrer entre 3,5 a 7 milhões de indivíduos em uma colônia adulta. Além disso, a densidade de colônias de A. sexdens rubropilosa pode ser superior a 50 formigueiros/ha, entre formigueiros novos e adultos, segundo Araújo et al. (1997) e Zanetti et al. (2003) em povoamentos de Eucalyptus spp.

Durante os 42 dias de avaliação, os formigueiros jovens produziram 10,40 g de resíduos e foram consumidos 14,28 $\mathrm{g}$ de folhas, e os formigueiros adultos, 64,33 g de resíduos e 103,12 $\mathrm{g}$ de folhas de $E$. grandis, respectivamente. $\mathrm{O}$ fator de conversão médio encontrado para os formigueiros de $A$. sexdens 
rubropilosa alimentados com E. grandis nessas condições de laboratório foi de 1,37 para os formigueiros jovens e de 1,60 para os formigueiros adultos. A média do fator de conversão entre os formigueiros adultos e jovens monitorados em laboratório foi de 1,49. Esse valor é próximo ao encontrado por Souto et al. (2007), que em seus experimentos encontraram fatores de conversão entre 1,45 e 1,63, porém difere dos dados encontrados por Autuori (1947), que encontrou um fator de conversão de 1,24.

Considerando a quantidade de folhas consumidas $(117,39 \mathrm{~g})$ e a quantidade de resíduo depositado (74,73 g) pelos formigueiros jovens e adultos, observou-se que $63,65 \%$ do material fornecido aos formigueiros converteram-se em resíduos. Esse valor corrobora Lugo et al. (1973), em cujos estudos $64,57 \%$ do material carregado para a colônia foi devolvido às panelas de resíduos. Pode-se observar que as saúvas não só devolvem os nutrientes retirados da vegetação por meio de herbivoria, como também fornecem um aporte de nutrientes, possivelmente oriundos da atividade de processamento realizado pelo fungo simbionte, quando analisados para vegetação do tipo cerrado (ABRIL; BUCHER, 2004). No campo, esse percentual de $63,65 \%$ poderia ser incorporado ao solo como matéria orgânica. Assim, considerando-se que no campo um formigueiro pode consumir 1 ton de folhas em 1 ano (AMANTE, 1967), aproximadamente $636,5 \mathrm{~kg}$ de resíduos poderiam ser incorporados ao solo.

\section{CONCLUSÕES}

- Tomando-se por base esses valores, podemos dizer que as colônias de formigas-cortadeiras são relevantes quando se trata de ciclagem de nutrientes sobre a vegetação adjacente, principalmente em locais com altos índices de infestação desses insetos, pois, apesar de cortarem as folhas das árvores, elas devolvem parte dos nutrientes ao solo, através da deposição do resíduo que geram.

\section{REFERÊNCIAS}

ABRIL, A. B.; BUCHER, E. H. Evidence that the fungus cultured by leaf-cutting ants does not metabolize cellulose. Ecology Letters, v. 5: 325 - 328. 2002. 247. 2004.

Nutritional sources of the fungus cultured by leaf-cutting ants. Applied Soil Ecology, 26: 243 -

AMANTE, E. Prejuízos causados pela formiga saúva em plantações de Eucalyptus e Pinus no Estado de São Paulo. Silvicultura em São Paulo, São Paulo, 6: 355 - 363. 1967.

ARAÚJO, M. S.; DELlA LUCIA, T. M. C.; MAYHÉ-NUNES, A. J. Levantamento de Attini (Hymenoptera: Formicidae) em povoamento de Eucalyptus na região de Paraopeba, Minas Gerais, Brasil. Revista Brasileira de Zoologia, 14: 323 - 328. 1997.

AUTUORI, M. Contribuição para o conhecimento da saúva (Atta spp. Hymenoptera, Formicidae).VI - O sauveiro depois da primeira revoada (Atta sexdens rubropilosa Forel, 1908). Arq. Inst. Biol., São Paulo, 18: 39 - 70. 1947.

FORTI, L. C.; CROCOMO, W. B.; GUASSU, C. M. O. Bioecologia e Controle das Formigas Cortadeiras de Folhas em Florestas Implantadas. São Paulo. FEPAF. Boletim didático n. 4. 30 p. 1987.

FORTI, L. C. Professor desvenda formigueiro. Pragas agrícolas. O Estado de São Paulo. Boletim didático. 17 de setembro de 2008.

FOWLER, H. G.; FORTI, L. C.; DI ROMAGNANO, L. F. T. Methods for the evaluation of leaf-cutting ant harvest. In: VANDER MEER, R. K.; JAFFE, K.; CEDENO, A. (Ed.). Applied Myrmecology: a world perspective. Boulder: Westview Press, 1990. p. 228 - 241.

JONKMAN, J. Determination of the vegetative material intake and refuse production ratio in two species of grass-cutting ants (Hym.: Attini). Zeitschrift für Angewandte Entomologie, v. 84, n. 1, p. 25 - 34. 1977.

LUGO, A. E.; FARNWORTH, E. G.; POOL, D.; JEREZ, P.; KAUFMAN, G. The impact of the leaf cutter ant Atta colombica on the energy flow of a tropical west forest. Ecology, 54: 1292 - 1301. 1973. 
MOUTINHO, P.; NEPSTAD, D. C.; DAVIDSON, E. A. Influence of leaf-cutting ant nests on secondary forest growth and soil properties in Amazônia. Ecology, 84: 1265 - 1276. 2003.

ROBBINS, C. T. Wildlife feeding and nutrition. San Diego: Academic Press, 1993. 352 p.

SILVA, F. A. S.; AZEVEDO, C. A. V. Versão do programa computacional Assistat para o sistema operacional Windows. R. Bras de Produtos Agroindustriais, Campina Grande, v. 4, n. 1, p. 71 - 78, 2002.

SOUTO, L. S.; GUERRA, M. B. B.; SCHOEREDER, J. H.; SHAEFER, C. E. G. R.; SILVA, W. L. da. Determinação do fator de conversão em colônias de Atta sexdens rubropilosa (HYMENOPTERA: FORMICIDAE) e sua relação com a qualidade do material vegetal cortado. Revista Árvore, v. 31, n. 1, p. 163 - 166. Viçosa, 2007.

VERCHOT, L. V.; MOUTINHO, P. R.; DAVIDSON, E. A. Leafcutting ant (Atta sexdens) and nutrient cycling: Deep soil inorganic nitrogen stocks, mineralization, and nitrification in Eastern Amazonia. Soil Biol. Biochem., 35: 1219 - 1222. 2003.

WEBER, N. A. A ten-year laboratory colony of Atta cephalotes. Annals of Entomological Society of America, v. 69, n. 6, p. 825 - 829. 1976.

ZANETTI, R.; ZANUNCIO, J. C.; MAYHÉ-NUNES, A. J.; MEDEIROS, A. G. B.; SOUZA-SILVA, A. Combate sistemático de formigas-cortadeiras com iscas granuladas em eucaliptais com cultivo mínimo. Revista Árvore, 27: 387 - 392. 2003. 
